\title{
SULLE VARIAZIONI MAGNETICHE PRODOTTE NEL NICHEL DALLE DEFORMAZIONI.
} G. ERCOLINI.

1. - La singolarità di alcuni fenomeni magneto-elastici presentati dal nichel $\mathrm{mi}$ ha fatto ritenere utile di ripetere con questo metallo delle esperienze simili a quelle che gia eseguii sul ferro posto in un campo magnetico alternativo ").

Invero, come pel ferro, le note e numerose esperienze eseguite fin qui col nichel riguardano propriamente l'influenza che le azioni elastiche esercitano sulla sua magnetizzazione irreversibile e niente, ch' io sappia, si conosce dei fenomeni che succedono quando l' isteresi magnetica sia totalmente o in parte ridotta. Se molto interessante risulta il primo studio, forse non lo è meno l' esame dell' altro caso come quello in cui, riducendo completamente l' isteresi, il numero delle variabili dei fenomeni che si producono viene così a diminuire, mentre poi è dato esaminare, nelle sue diverse fasi, la perturbazione causata dalla isteresi stessa, quando questa sia grado a grado ridotta.

E anche pel nichel è prevedibile che l' eliminazione del$l^{\prime}$ isteresi magnetica porti a risultati semplici.

I fili di nichel che ho adoperato, del diametro di cm. 0,1, sono costantemente lunghi $50 \mathrm{~cm}$. e vengon posti, come già. quelli di ferro, nell' interno d'una doppia spirale verticale, lunga $80 \mathrm{~cm}$., necessaria per sovrapporre ad uno cóstante $\mathrm{H}_{\mathrm{t}}$ un campo alternato $H_{2}$ della stessa direzione, avendosi in tal modo un campo risultante alternativo simmettrico rispetto al valore di $\mathrm{H}_{1}$.

1) Nuovo Cim., novembre 1910 , 
Quando un filo di nichel si trova in tal campo assai intenso, od anche in uno alternatiro simmetrico rispetto a zero, si avverte un fenomeno che non notai col ferro: il filo si mette in intensa vibrazione longitudinale e si genera un suono cha varia $d$ ' intensità al mutare del valore del campo e del peso tensore. Questo fenomeno, dovuto a magnetostrizione ${ }^{1}$ ), non è qui da riguardarsi come una causa perturbatrice, poichè, com'è noto, le vibrazioni e gli sçuotimenti hanno la proprieta di ridurre l'isteresi magnetica: per quest' effetto all' azione del campo alternativo vien quindi a sovrapnorsi favorevolmente quella delle vibrazioni longitudinali.

Ho studiato fili ricotti con un' intensa corrente elettrica e fili crudi; pei primi ho trovato che $\mathrm{i}$ fenomeni che si producono dipendono, in intensità e spesso anche in senso, dal grado di ricottura. Non la grande varietà di risultati ottenuti descriverò minutamente, ma, per brevità, riporterò solo i fatti principali e tipici che ho osservato nel corso delle numerosissime esperienze.

Pei necessari confronti ho dovuto ripetere l' esame dei fenomeni che si producono in campi magnetici costanti ed ho anche qui osservato alcuni fatti, non ancora avvertiti, che esporrò brevemente.

\section{Trazione.}

2. - Fili ricotti. - Prima di cominciare le esperienze ogni filo viene smagnetizzato sottoponendolo più volte al campo alternativo di 50,7 uniti c. g. s. che si fid decrescere lentamente fino a zero.

Che i risultati forniti dalla trazione dipendono dal grado di ricottura del nichel lo mostrano $i$ seguenti risultati offerti da fili cimentati nel solo campo terrestre. La seconda colonna della tabella porta le deviazioni del magnetometro ottenute stirando un filo ricotto al rosso vivo con una corrente di 23 amp. mantenuta per $120^{\mathrm{s}}$ e diminuita poi lentamente; la terza colonna dà i risultati con un filo ricotto con corrente

4) Dell' influenza d' un campo alternato sulla magnetostrizione riferirò in altra occasione. 
di 24 amp. per $20^{\mathrm{s}}$; la quarta quelli avuti con altro filo portato al rosso scuro da una corrente di $20 \mathrm{amp}$. che circola per $\check{\partial}^{\mathrm{s}}$; l' ultima quelli che dà un filo ricotto due volte al rosso scuro per $\tilde{\partial}^{\text {s }}$ con una corrente di $20 \mathrm{amp}$. In ogni caso il carico iniziale, preso come zero, da cui si comincia la trazione è di $1 \mathrm{Kg}$.

$$
\begin{array}{l|r|r|r|r}
\text { Kg. } & & & & \\
0 & 0.0 & 0.0 & 0.0 & 0.0 \\
1 & -22.0 & -39.0 & -2.0 & -28.5 \\
2 & -77.5 & -97.5 & -5.5 & -67.0 \\
3 & -108.5 & -143.0 & -8.5 & -99.0 \\
4 & -132.0 & -171.0 & -13.5 & -121.5 \\
5 & -140.5 & -185.5 & -18.0 & -136.5 \\
6 & -146.0 & -199.5 & -24.5 & -156.0 \\
7 & -151.0 & -203.5 & -31.0 & -164.5 \\
8 & -154.5 & -203.0 & -40.5 & -170.0
\end{array}
$$

Molta, come si vede, è l' influenza esercitata dalla grandezza della temperatura di ricuocimento e dalla durata della sua azione sulle diminuzioni di magnetizzazione provocate da uno stesso peso tensore e percio mi sono limitatato ad esaminare dei fili trattati sempre nello stesso modo.

Sotto la tensione costante di $1 \mathrm{Kg}$. uno di questi fili, portato in precedenza al rosso vivo con la corrente di 23 a. per $180^{\mathrm{s}}$ e annullata poi gradatamente in $300^{\mathrm{s}}$, presenta un ciclo magnetico, fissato fra $i$ valori estremi $H_{1}= \pm 12.2$ unità c. g. s., e una curva di magnetizzazione anisteretica molto simili a quelli del ferro; quest' ultima curva parte ciò̀ da zero e traversa il ciclo da cui esce al di sopra verso i valori più grandi di $\mathrm{H}_{1}$. Sottoposto, in campo costante, alla trazione graduale di altri $5 \mathrm{Kg}$. partendo dalla curva di prima magnetizzazione, si ottiene un ciclo magneto-elastico che si fissa assai presto innalzandosi verso la curva di magnetizza- 
zione anisteretica, come lo mostrano questi risultati pel campo $\mathrm{H}_{1}=1$ unità c. g. s.

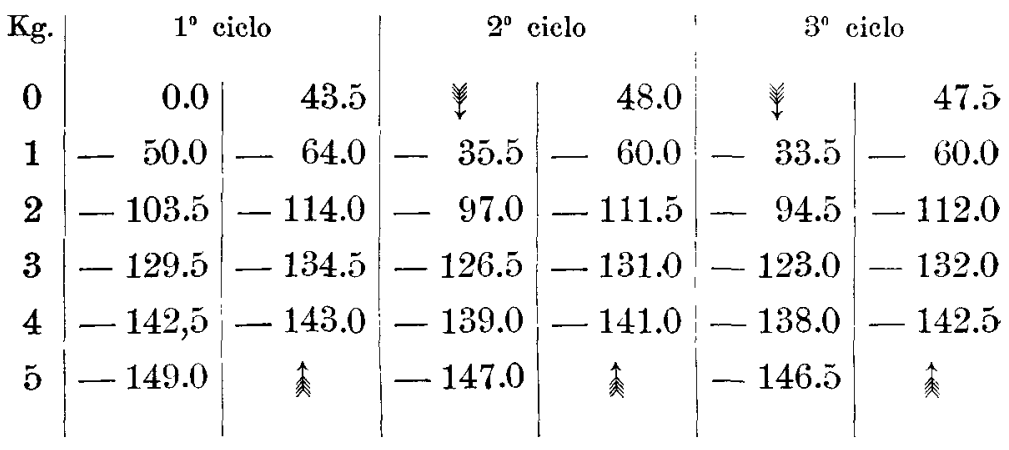

Ma aumentando il valore del campo $H_{1}$, e perciò avvicinandosi alla curva anisteretica, l' innalzamento del ciclo diventa sempre più piccolo.

3. - Se la trazione si opera partendo dalla curva di magnetizzazione anisteretica, le diminuzioni del momento prodotte dalla tensione aumentano e il rilasciamento non porta mai a valori positivi della magnetizzazione. Infatti pel campo $\mathrm{H}_{\mathrm{i}}=1$ unità si ottiene:

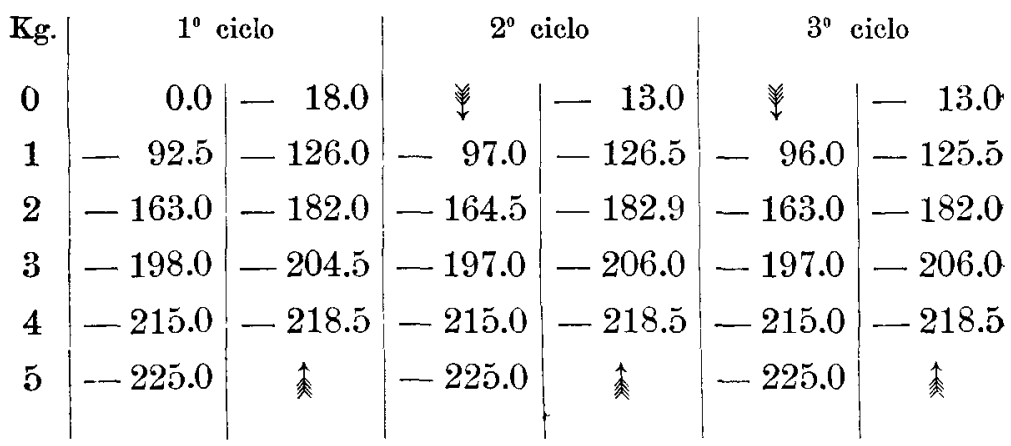

Gli stessi fatti succsdono per tutti i valori del campo $\mathrm{H}_{1}$ : $\mathrm{i}$ cicli magneto-elastici fissati presentano una forma più allungata e un' area un po' più grande di quelle che essi hanno quando si produce la trazione sulla curva di prima magnetizzazione. 
4. - Risultati vari e interessanti si hanno se si fa agire il peso tensore sul filo magneticamente ciclizzato. Anche qui, come nel ferro, se si parte da punti del ciclo magnetico posti al di sotto della curva anisteretica si hanno con la trazione effetti diversi, per lo stesso valore del campo $\mathrm{H}_{4}$, da quelli prodotti partendo da punti al di sopra di quella curva e l'entità delle differenze che si notano dipende dal valore del campo $H_{1}$, cioè dall' essere con questo più o meno distanti dalla curva di magnetizzazione reversibile.

Ciclizzato il filo fra $\mathrm{i}$ valori estremi $\mathrm{H}_{1}= \pm 12,2$ unità, ci si arresta al valore del campo magnetico terrestre, ma una volta al di sotto della curva anisteretica, poi al di sopra: la trazione dà rispettivamente $i$ risultati a sinistra e a destra scritti, pei primi due cicli, in questa tabella:

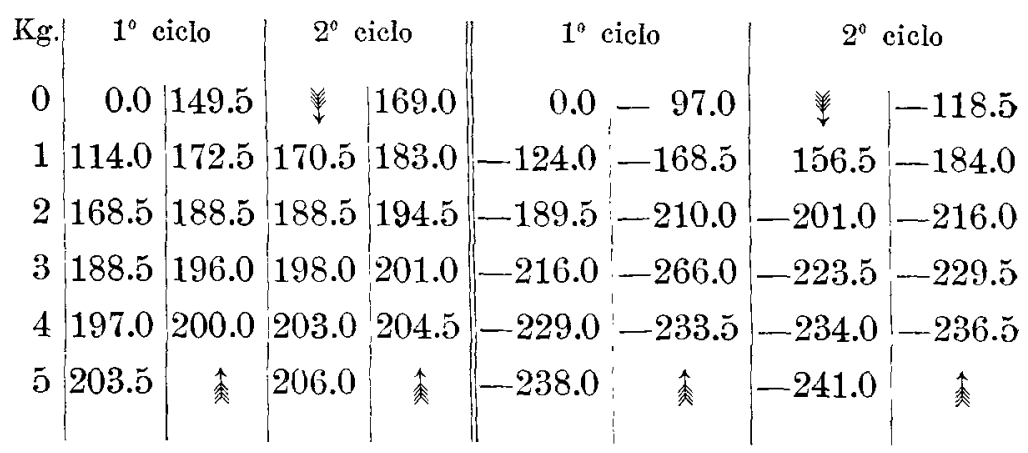

Nel primo caso la trazione produce un aumento di magnetizzazione e il rilaseiamento una diminuzione, nel secondo i fenomeni s' invertono; in entrambi la ripetizione del ciclo elastico avvicina il punto rappresentativo della magnetizzazione alla curva anisteretica.

Se il valore del campo $H_{1}$ aumenta, la prima trazione può produrre degli effetti singolari. Cosi, arrestandosi nella ciclizzazione al valore $H_{t}=1$ unita, ma al di sotto della curva di magnetizzazione reversibile, si ottiene: 


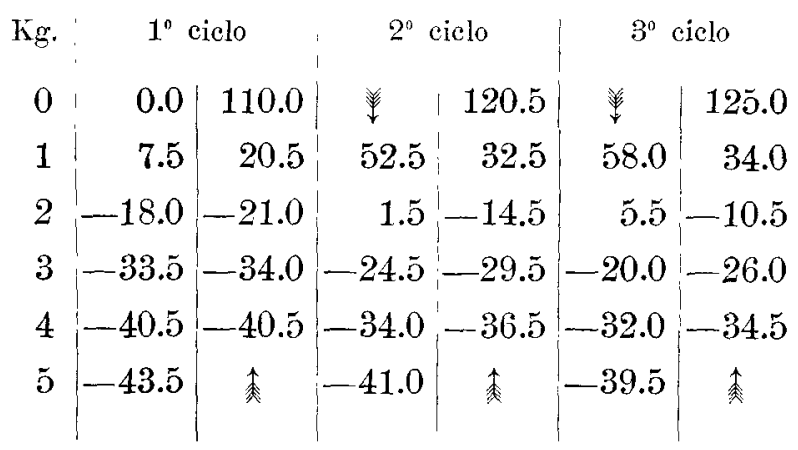

Qui la trazione produce da prima un aumento di magnetizzazione, ma le successive tensioni danno sempre una diminuzione. Però il fenomeno generato dalla prima trazione non si verifica se il valore del campo cresce. Quindi, considerando gli effetti generati dalle trazioni successive alla prima, possiamo concludere che " nei fili magneticamente eiclizzati la trazione produce sempre diminuzione di magnetizzazione e il rilasciamento sempre aumento quando il campo magnetico costante non è troppo debole (di 1 o più unità), mentre in campi molto piccoli (campo terrestre) succede lo stesso se si parte da punti del ciclo magnetico posti al di sopra della curva di magnetizzazione anisteretica, e avviene il rovescio movendosi da punti posti al di sotto di quella curva. In ogni caso il ciclo magneto-elastico va a fissarsi verso la curva anisteretica ».

כ. - L'azione d'un campo alternato $\mathrm{H}_{2}$ sulle variazioni magnetiche prodotte dalla trazione dipende dal valore del campo costante $\mathrm{H}_{1}$ e da quello $\mathrm{H}_{3}$. Se $\mathrm{H}_{1}$ è debole, un piccolo campo $\mathrm{H}_{2}$ basta per rendere quelle variazioni considerevolmente minori: così quando $\mathrm{H}_{4} \dot{e}$ il campo terrestre e $\mathrm{H}_{2}=0.9$ unità si ottengono i risultati a sinistra della tabella, ove gli altri son quelli che si hanno col solo $\mathrm{H}_{1}$. 


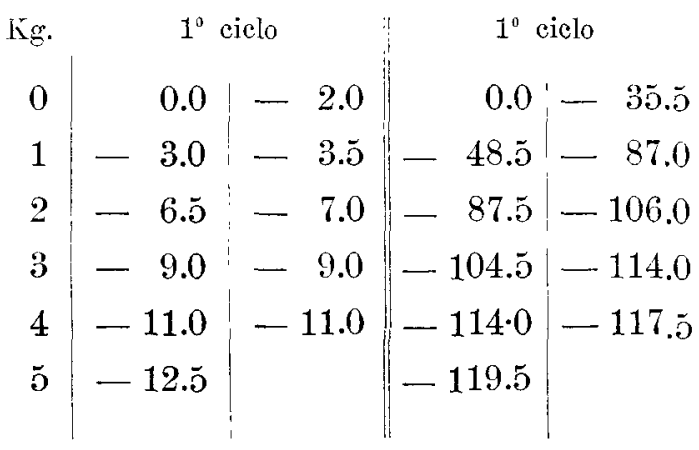

Le variazioni per trazione decrescono ancora quando $\mathrm{H}_{2}$ aumenta d'intensità e risultano già trascurabili allorchè $\mathrm{H}_{2}=20,1$ unità.

Se $\mathrm{H}_{4}$ cresce d'intensità, le riduzioni operate da un dato $\mathrm{H}_{2}$ si fanno meno grandi, ma poi al crescer di $\mathrm{H}_{2}$ esse diminuiscono e col valore di $\mathrm{H}_{2}$. più alto si ottengono solo delle molto piccolo diminuzioni di magnetizzazione.

Tale comportamento si ha pure con campi $\mathrm{H}_{4}$ più intensi; in tal caso facendo $\mathrm{H}_{2}=50.7$ unità le diminuzioni che si ottengono sono ancora considerevoli, come lo provano questi risultati : 


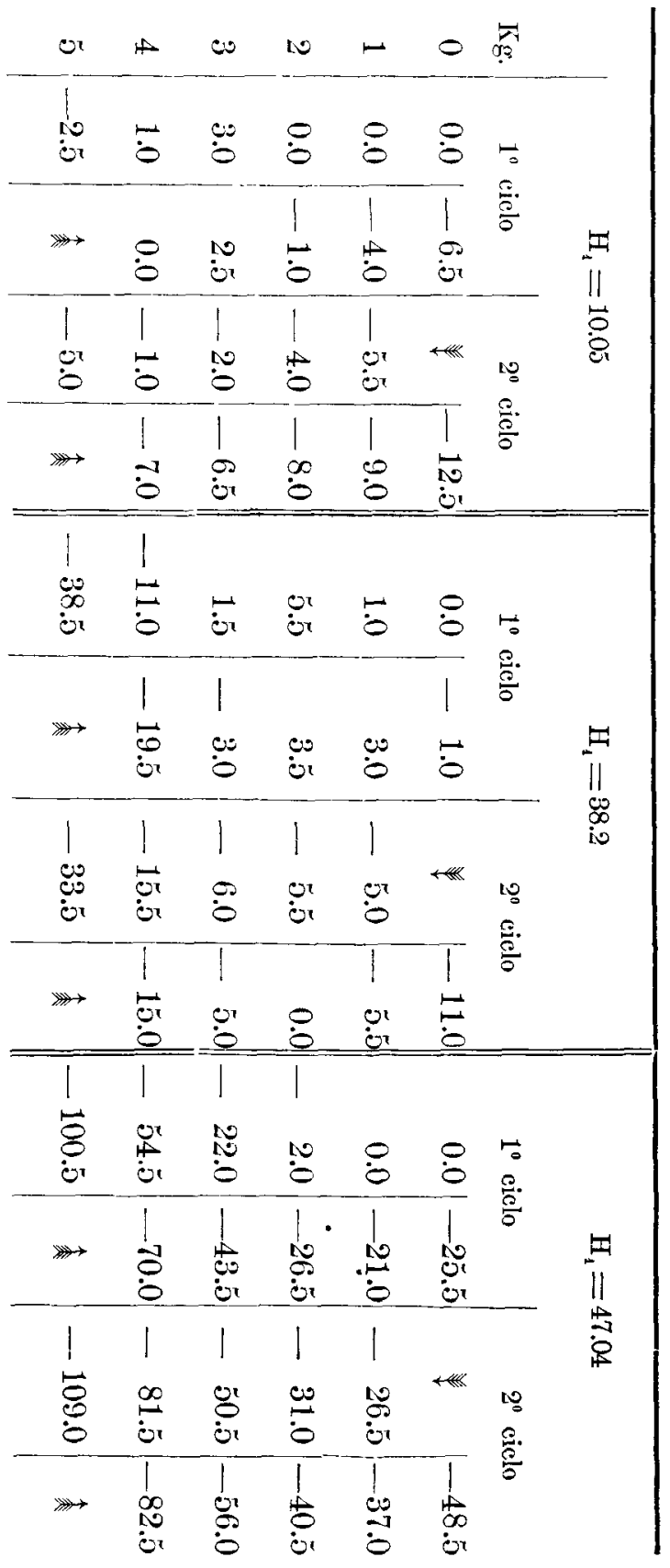


Si nota che la trazione causa in generale da principio un piccolo aumento di magnetizzazione seguito da più grande diminuzione e il rilasciamento produce effetti inversi. Le curve magneto-elastiche presentano ora la loro convessità all' asse dei carichi, al rovescio di quel che accade in campi costanti e in quelli deboli alternati.

Ma l'andamento generale dei fenomeni mostra che le variazioni magnetiche ottenute con campi $H_{4}$ intensi finirebbero probabilmente per scomparire, se l' intensità del campo alternato ad essi sovrapposto fosse maggiore di quella massima ( 50,7 uniti) che ho potuto avere per un tempo lungo.

Possiamo concludere che «quando il campo alternato ò sufficiente a ridurre completamente l'isteresi magnetica, le variazioni di magnetismo longitudinale prodotte ordinariamente dalla trazione nel nichel ricotto scompaiono: essa non ha più sensibile influenza ».

6. - Fili crudi. - Per portare questi fili in uno stato magnetico ben definito occorre fare agire su di essi un forte campo alternato decrescente: l' intensità iniziale di questo del valore di 50.7 unità non é sufficiente per produrre risultati regolari ed ottenere dei cicli magnetici ben definiti. Ho perciò dovuto crescere fino a circa 80 unità l'intensità iniziale del campo alternato smagnetizzante per ottenere resultati concludenti.

I fili di nichel molto crudi che ho adoperato, assoggettati in principio a questo trattamento, sotto la tensione iniziale costante di $4 \mathrm{Kg}$. necessaria a tenerli diritti, presentano dei cicli magnetici, fra $i$ valori estremi $H_{1}= \pm 12.2$ unità, molto stretti e prossimi all' asse del campo $\mathrm{H}_{3}$, mentre poi danno una curva di magnetizzazione anisteretica molto rilevata e simile a quella dei fili ricotti. Ciò è mostrato dalla seguente tabella, nella prima colonna della quale son riportati $i$ valori del campo $H_{1}$, nelle due successive le deviazioni del magnetometro quando il ciclo magnetico è fissato, nelle altre due le deviazioni ottenute per valori negativi di $\mathbf{H}_{\mathbf{1}}$; 
nell' ultima i valori assunti dalle doriazioni, nel solo caso di $\mathrm{H}_{1}$ positivo, dopo di aver ridotto la isteresi magnetica ${ }^{1}$ ).

\begin{tabular}{c|r|r|r|r|r}
$\mathrm{H}_{1}$ & & & & & \\
0.0 & 7.5 & -6.5 & F & 7.5 & 0.0 \\
1.0 & 12.0 & -1.5 & -11.5 & 2.0 & 105.0 \\
4.0 & 20.0 & 7.5 & -19.0 & -7.0 & 185.5 \\
7.0 & 27.5 & 21.0 & -27.0 & -19.0 & 228.5 \\
10.05 & 31.0 & 22.5 & -28.5 & -26.0 & 260.5 \\
12.2 & 36.5 & 疋 & -33.5 & 柰 & 283.5
\end{tabular}

Come si vede nel nichel crudo che ho adoprato il ciclo magnetico è percorso nello stesso senso degli indici d' un orologio, è ciò̀ ad isteresi negativa, al contrario di quelìo presentato dal ferro, e nella regione positiva del piano $H_{4}$ (ascisse) - momento magnetico si trova al di sotto della curra anisteretica che lo traversa in una regione limitatissima, molto prossima a $\mathrm{H}_{1}=0$.

Riguardo alle variazioni prodotte dalla trazione in campo $H_{1}$ costante, lungo la eurva di prima magnetizzazione, risulta che il ciclo magneto-elastico si fissa tutto al di. sotto dell'ascissa $\left(H_{1}\right)$ se il campo è debole (una unità o meno), ma per valori più grandi di $H_{1}$ esso taglia l'asse $H_{1}$; mentre poi il ciclo che si esiguisce partendo dalla curva anisteretica si fissa tutto in basso e comprende sempre un'area maggiore di quella che esso ha nel primo caso.

Tutto ciò è mostrato dai dati delle seguenti tabelle: a sinistra i risultati sono ottenuti con la trazione da 4 a $16 \mathrm{Kg}$. partendo dalla curva di prima magnetizzazione; gli altri si hanno movendosi dalla curva anisteretica.

1) Questi ultimi valori ottanuti a campo $H_{4}$ crescente non coincidono esattamente con quelli a campo decrescente quando $H_{1} \dot{e}$ intenso, ma le differanze, sono piccole; sicchò si puó ritenere che la vera curva anisteretica differisea di poco da quella che si ha per l'azione di $\mathrm{H}_{2}=50.7 \mathrm{u}$ nità. Adoperando infatti il campo alternato più intenso, quelle differenze scompaiono e la curva anisteretica si mostra un po' più elevata dell'altra. 
VARIAZIONI MAGNETICHE ECC.

$$
\mathrm{H}_{1}=1.0
$$

\begin{tabular}{|c|c|c|c|c|c|c|c|}
\hline $\mathrm{Kg}$. & $1^{\circ}$ ciclo & $2^{0} \mathrm{c}$ & iclo & $1^{0} \mathrm{e}$ & iclo & $2^{\circ} \mathrm{c}$ & ciclo \\
\hline 0 & $0.0-1.5$ & 亲 & -1.0 & 0.0 & -10.5 & 戠 & -12.5 \\
\hline 2 & $-1.0-5.0$ & -2.0 & -5.0 & -7.5 & -23.0 & $-16 . \tilde{0}$ & -25.5 \\
\hline 4 & $-3.5-8 . \tilde{0}$ & -4.0 & -8.0 & -17.0 & -34.0 & -26.0 & -37.5 \\
\hline 6 & $-5.0-12.0$ & -8.0 & -20.0 & -29.5 & -47.0 & -36.5 & -48.0 \\
\hline 8 & -9.5 .15 .5 & $-11 . \tilde{0}$ & -16.0 & -43.5 & -57.5 & -48.0 & -58.5 \\
\hline 10 & $-15.0-18.5$ & -16.5 & $-18 . \tilde{0}$ & -57.0 & -65.0 & -60.0 & -66.5 \\
\hline 19 & -19.5 & -20.5 & 类 & -71.5 & 本 & -71.0 & $\hat{\lambda}$ \\
\hline
\end{tabular}

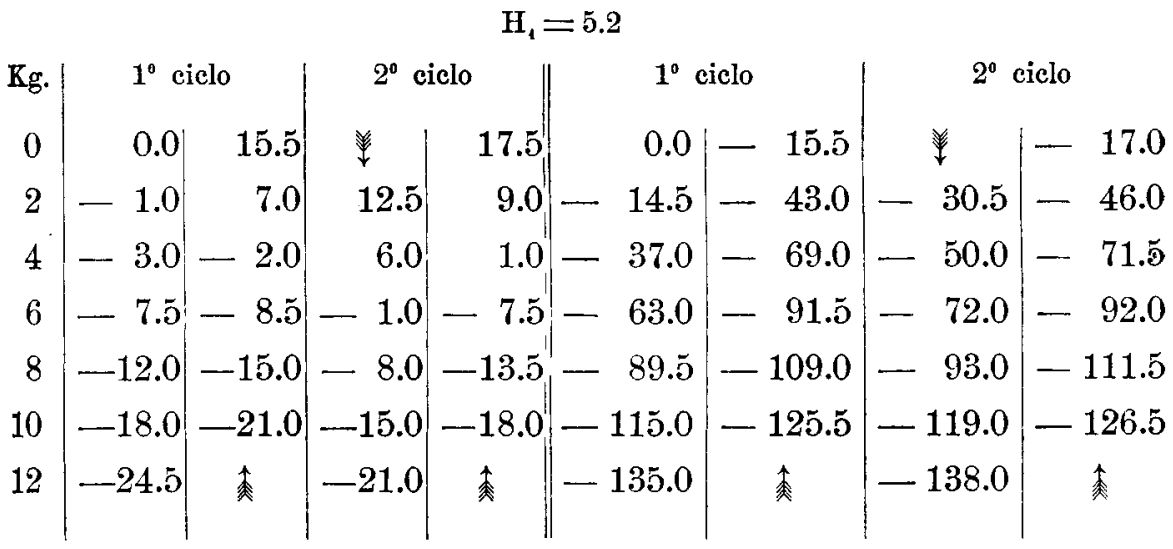

7. - Singolare è il comportamento dei fili magneticamente ciclizzati, poichè si riscontra che nonostante, come abbiamo visto, il ciclo magnetico sia al di sotto della curva anisteretica, gli effetti della trazione son diversi a seconda, per lo stesso valore del campo, del punto del ciclo magnetico da cui la si inizia. Infatti : un filo crudo è ciclizzato fra i valori estremi $H_{1}= \pm 12.2$ unità; al valore $H_{1}=5,2$ unità si produce la trazione, ma una volta si comincia dal punto del ciclo più elevato, un'altra dal punto più basso rispetto all'ascissa $\mathrm{H}_{1}$; si ottengono $\mathrm{i}$ risultati segnati rispettivamente a sinistra e a destra della tabella che segue: 


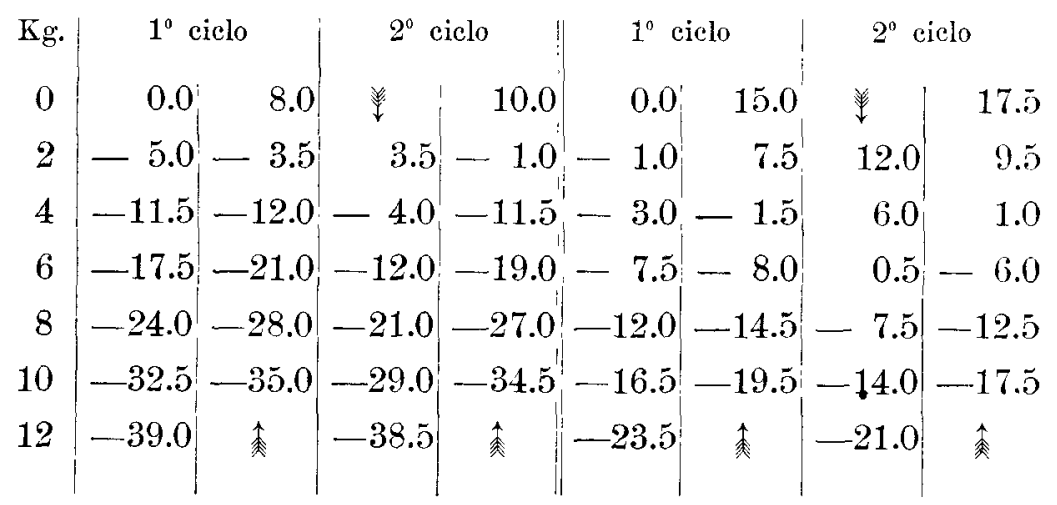

Dal confronto di questi risultati con quelli dell' ultima tabella del $\S$ precedente si ricava che, se si parte con la trazione dal punto più elevato del ciclo magnetico, si hanno diminuzioni più grandi che movendosi dalla curva di prima magnetizzazione: il ciclo magneto-elastico e più basso rispetto all' ascissa $\mathrm{H}_{1}$. Nell' altro caso questo è quasi perfettamente uguale a quello ottenuto sulla curva di prima magnetizzazione.

In ogni campo gli effetti prodotti dalla trazione, per lo stesso valore di esso, sono diversi a seconda del punto del ciclo magnetico da cui si comincia la tensione, ma i fatti non sono ora più da riferirsi alla curva di magnetizzazione anisteretica verso la quale il ciclo magneto-elastico si avvicina, di poco in generale, in un caso, ma se ne allontana invece, e assai di più, nell' altro, quando ciò̀ col campo $H_{1}$ ci si arresta a punti. a cui si perviene con valori decrescenti di esso.

8. - Con la riduzione parziale d'isteresi magnetica, mediante un campo alternato $\mathrm{H}_{2}$ non molto intenso sovrapposto a quello costante $\mathrm{H}_{1}$, la trazione può dare effetti molto singolari. La fig. 1 mostra le curve dei cicli che si ottengono col campo $\mathrm{H}_{3}=20,1$ unità: la $\mathrm{I}$ è ottenuta col campo $\mathrm{H}_{1}=1$ unità, nella II ̀̀ $H_{1}=5,2$ unità e nella III è $\mathrm{H}_{1}=10,05$ unità c. g. s. In ogni caso la prima trazione produce da principio un aumento d'intensità di magnetizzazione a cui fa seguito una diminuzione, che diventa più cospicua maggiore $\grave{e}$ 
l'intensita di $\mathrm{H}_{1}$. In II pure la seconda trazione produce Io stesso effetto, mentre in III provoca sempre diminuzione.

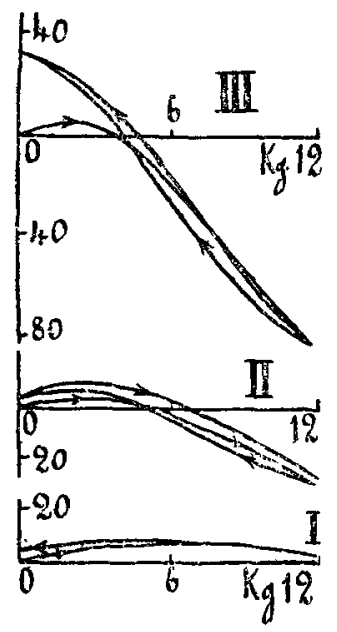

Fig. 1.

Ma anche col nichel crudo assoggettato al campo $\mathrm{H}_{2}=50,7$ unita la trazione non genera che diminuzioni insensibili o piccolissime, che diventano apprezzabili quando $H_{4}$ è assai in-

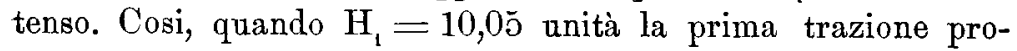
duce una diminuzione totale del momento magnetico corrispondente a 6 divisione della scala.

Concludendo si ha che nel nichel crudo le variazioni prodotte dalla trazione sono, a parità di condizioni, assai minori che in quello ricotto $\mathrm{e}$ in entrambi decrescono moltissimo, riducendosi a piccole diminuzioni di magnetizzazione, quando la isteresi magnetica è prossima a scomparire.

\section{Torsione.}

9. - L'influenza della torsione sul magnetismo del nichel dipende molto dalla tensione e dal campo magnetico a cui esso è assoggettato. 
Il Nagaoka trovò ') che sottoponendo il nichel a carichi tensori, crescenti con la grandezza del campo magnetico, alla diminuzione di magnetizzazione che si ottiene ritornando a torsione nulla $f a$ seguito una magnetizzazione inversa se si torce in senso contrario al primitivo, in modo che, quando lo stiramento sia sufficiente, le curve dei cicli magneto-elastici rassomigliano a quelle dei cicli d' isteresi magnetica, con 'la differenza però che risultano tracciate in senso inverso a queste, cioè son percorse nel senso del moto degli indici d'un orologio e si ha isteresi negativa. In certe condizioni di campo e di tensione l'asse della curva può anche girare di circa $90^{\circ}$.

Un bello studio intorno a questi fenomeni venne fatto, alcuni anni or sono, dal Prof. Cantone ${ }^{2}$ ). Io ho cercato come essi si modificano sotto $l^{\prime}$ influenza d' un campo alternato, ma esporrò brevemente da prima i risultati che si ottengono con piccola tensione e con campi non molto deboli, quando cioè non si produce il fenomeno Nagaoka.

10. - Fili ricotti. - Anche per la torsione gli effetti che questa produce dipendono dal grado di ricottura dei fili, nel senso che varia la grandezza della torsione che bisogna raggiungere perchè la magnetizzazione cessi di aumentare $e$ finisca per decrescere. Perciò mi son limitato all' esame dei fili ricotti, come per la trazione, con la corrente di 23 amp. per $180^{\mathrm{s}}$, producendo torsioni di poco superiori a $1^{\circ}$ per $\mathrm{cm}$. Assoggettando di questi fili al carico costante di $2 \mathrm{Kg}$. con torsioni $\alpha$ massime di $\pm 60^{\circ}$, a partire dalla curva di prima magnetizzazione, se il campo magnetico è di una o più unita, si ottengono le note curve di magnetizzazione a due cappi analoghe a quelle del ferro ma rovesciate: i cicli magneto-elastici si fissano allontanandosi dall' origine per avvicinarsi, al solito, alla curva di magnetizzazione anisteretica.

Se invece s'inizia la torsione partendo da questa curva il ciclo magnetico-elastico si fissa più prossimamente all' origine come mostrano $\mathrm{i}$ dati della seguente tabella, ove, a sini-

1) Phil. Mag., 27, 1889 e Wied. Ann., 53, 1894.

2) Nuovo Cim., 5, 1897. 
stra, si trovano le deviazioni del magnetometro prodotte dalla torsione, di $20^{\circ}$ in $20^{\circ}$, col campo $\mathrm{H}_{1}=1$ unità partendo dalla curva di prima magnetizzazione e a destra quelle avute iniziando la torsione dalla curva anisteretica, coll' avvertenza che le seconde colonne corrispondono in ogni caso a torsioni negative.

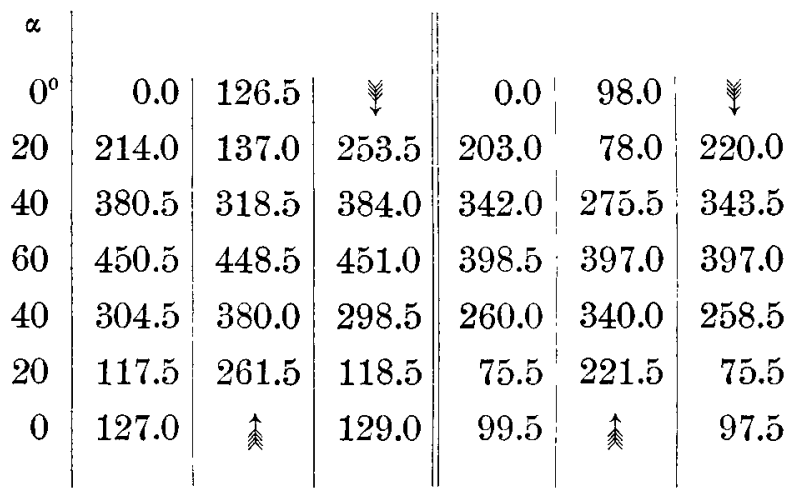

Nei fili magneticamente ciclizzati cominciando la torsione da punti al di sopra o al di sotto della curva anisteretica, il ciclo magneto-elastico si abbassa o s'innalza, come mostra la seguente tabella in cui nelle due colonne a sinistra, si hanno i principali risultati del primo e, nelle due seguenti, quelli dell' atro caso.

\begin{tabular}{|c|c|c|c|c|c|c|}
\hline $0^{\circ}$ & 0.0 & $20 . \check{\partial}$ & 0.0 & 246.0 & 0.0 & -2.0 \\
\hline 20 & 122.0 & 4.0 & 309.0 & 239.5 & -2.0 & -3.0 \\
\hline 40 & 259.5 & 165.5 & 498.气 & 430.0 & -9.0 & -7.0 \\
\hline 60 & 321.5 & 320.5 & 565.5 & 561.5 & -12.5 & -12.0 \\
\hline 10 & 162.0 & 260.0 & 429.0 & $492 . \check{\partial}$ & -6.0 & -7.5 \\
\hline 20 & 2.0 & 146.0 & 240.0 & 379.0 & -4.5 & -3.0 \\
\hline 0 & $21 . \bar{\jmath}$ & 榲 & 247.5 & 疋 & -1.0 & 本 \\
\hline
\end{tabular}

Coll' azione del campo alternato $\mathrm{H}_{2}$ tutte queste variazioni si riducono molto, finchè con $\mathrm{H}_{2}=50.7$ unità la tor- 
sione provoca delle variazioni insignificanti o soltanto delle piccole diminuzioni di magnetizzazione. Così con $\mathrm{H}_{1}=10.05$ unità e $\mathrm{H}_{2}=50.7$ unità c. g. s. si hanno i risultati scritti nelle due ultime colonne della tabella precedente,

11. - Fili crudi. - In questi le torsioni e detorsioni producono da prima un graduale aumento di magnetizzazione, che diminuisce considerevolmente quando si opera in campo alternato e non si hanno che lievi diminuzioni di magnetizzazione se $\mathrm{H}_{2}=50.7$ unità.

12. - Influenza della tensione. - Fili ricotti. - Se il campo costante è molto debole anche sotto la tensione di $2 \mathrm{Kg}$. si verifica il fenomeno del Nagaoka. Infatti nel solo. campo terrestre si ottiene:



L' esame di questi risultati mostra che le diminuzioni causate dalla detorsione sono maggiori degli aumenti prodotti dalla prima torsione: il ciclo taglia l' asse del campo e non è simmetrico rispetto all'origine, ma tende a diventarlo con la ripetizione, chè diminuiscono gli aumenti al massimo di torsione nel primo senso e aumentano quelli di segno contrario al massimo di torsione nell'altro senso. Il ciclo è percosso nel verso del moto degli indici d' un orologio e si ha perciò isteresi negativa.

Considerevoli spostamenti del ciclo si ottengono se si ciclizza il filo e si opera la torsione partendo al di sopra o al di sotto della curva anisteretica. Nei due casi si hanno questi risultati segnati rispettivamente a sinistra e a destra: 


\begin{tabular}{|c|c|c|c|c|c|c|c|c|}
\hline$\alpha$ & \multicolumn{2}{|c|}{$1^{0}$ cielo } & \multicolumn{2}{|c|}{$2^{\circ}$ eiclo } & \multicolumn{2}{|c|}{$1^{0}$ ciclo } & \multicolumn{2}{|c|}{$2^{0}$ ciclo } \\
\hline $0^{\circ}$ & 0.0 & -139.0 & 呈 & -136.0 & 0.0 & 152.5 &  & 152.0 \\
\hline 20 & 55.0 & -229.0 & -22.0 & -238.5 & -41.5 & 278.5 & $67 . \tilde{\delta}$ & 270.0 \\
\hline 40 & $116 . \overline{0}$ & -309.0 & 60.0 & $-314 . \check{0}$ & -52.0 & 418.0 & 44.0 & 404.0 \\
\hline 60 & 170.5 & -361.0 & 115.5 & -357.0 & -18.0 & 497.5 & 72.0 & 478.5 \\
\hline 40 & 10.0 & -374.0 & -11.5 & -372.5 & 84.0 & 482.0 & 141.0 & 466.5 \\
\hline 20 & -181.0 & -344.5 & -196.0 & -353.0 & 242.5 & 442.0 & 253.5 & 429.0 \\
\hline 0 & -290.0 & 委 & -300.0 & 乘 & 361.0 & 奏 & 353.5 & 本 \\
\hline
\end{tabular}

Quando la torsione è iniziata movendo al di sopra della curva anisteretica la prima torsione provoca un aumento di magnetizzazione, ma la detorsione dà una grandissima diminuzione che seguita per buona parte della torsione in senso inverso. In complesso il ciclo, $\dot{e}$, rispetto a quello precedentemente esaminato, molto spostato al di sotto dell'origine $e$ presenta un' area maggiore.

$\mathrm{Nel}$ secondo caso la torsione provoca da prima una $d i$ minuzione di magnetizzazione poi un aumento più piccolo, ma la detorsione l' accresce in tal modo che il ciclo è ora tutto al di sopra, e assai discosto dall'origine, e presenta pure un' area maggiore di quello esaminato per primo. Come questo $\mathrm{i}$ due ultimi cicli sono ad isteresi negativa.

Risultati ancor più cospicui si hanno quando si aumenta la torsione. Con un filo torto di $\pm 220^{\circ}$ ho avuto $\mathrm{i}$ risultati rappresentati nella fig. 2. La $\mathrm{I}$ è $\overline{\text { ll }}$ ciclo che nel campo terrestre e con la tensione di $2 \mathrm{Kg}$. si ha dal filo non ciclizzato; la II è il ciclo ottenuto con ciclizzazione e partenza, per lo stesso campo terrestre, dal punto al di sopra della. curva anisteretica; la III corrisponde all' altro caso. Se s'immaginano gli assi delle tre figure trasportati sopra una stessa. retta, si nota subito quali enormi spostamenti dei cicli posson generarsi nei vari casi, quali notevoli variazioni si hanno nell' area da essi abbracciata e «quali grandi magnetizzazioni juò destare l' azione elastica ».

Elevando, di 2 in $2 \mathrm{Kg}$., il peso tensore, i risultati precedenti, ottenuti con torsioni di $\pm 60^{\circ}$, in principio non va- 
riano molto, ma poi l'area del ciclo magneto-elastico che si ha cominciando la torsione dalla curva di prima magnetizzazione è sempre assai rilevante, mentre diminuisce quella dei cicli ottenuti dopo d'aver ciclizzato magneticamente.

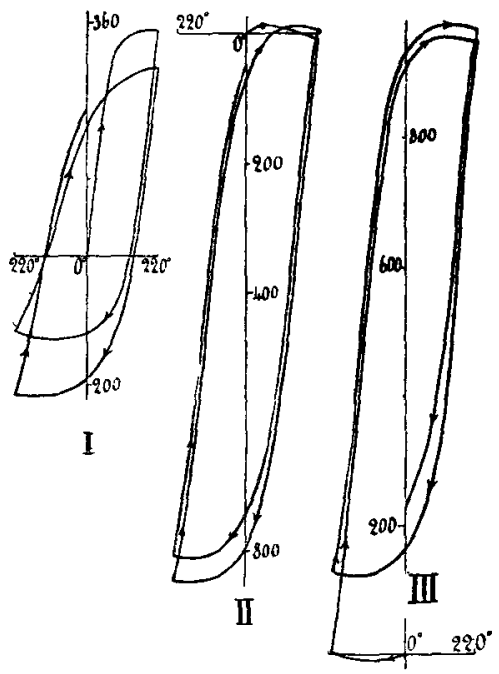

Fig. 2.

Passando col carico tensore da 8 a $10 \mathrm{Kg}$. il filo subisce un allungamento permanente di $1,5 \%$; la torsione in campo terrestre produce ora diminuzione di magnetizzazione e la detorsione, con la conseguente torsione in senso inverso, danno aumento, diguisachè l' asse del ciclo si trova orientato diversamente che nei casi precedenti; inoltre il ciclo stesso è a isteresi positiva. Infatti si ha:

\begin{tabular}{|c|c|c|c|c|c|}
\hline$\alpha$ & \multicolumn{2}{|c|}{$1^{0}$ ciclo } & \multicolumn{3}{|c|}{$2^{n}$ ciclo } \\
\hline $0^{\circ}$ & 0.0 & $-\quad 4.0$ & 事 & - & 4.0 \\
\hline 20 & -4.5 & 3.0 & $-\quad 9.0$ & & 3.0 \\
\hline 40 & -7.0 & 10.0 & -12.0 & & 1.0 \\
\hline 60 & -9.0 & 17.5 & -15.0 & & 18.5 \\
\hline 40 & $-\tilde{\mathbf{0}} .0$ & 14.0 & -9.0 & & 14.5 \\
\hline 2 & $-1 . \overline{2}$ & 9.0 & $-\quad 2.0$ & & 10.0 \\
\hline 0 & 5.0 & 乘 & 4.0 & & 秀 \\
\hline
\end{tabular}


Con tensioni crescenti aumenta leggermente il valore del campo magnetico con cui può prodursi il fenomeno Nagaoka; $\mathrm{ma}$ in ogni caso tutte le variazioni, generate dalla torsione con campi $H_{1}$ di piccola intensità e carichi differenti, scompaiono se al campo costante si sovrappone quello alternato $\mathrm{H}_{2}=50.7$ unità c. g. s. Possiamo quindi ritenere che «il fenomeno del Nagaoka è dovuto a variazioni di isteresi magnetica provocate dalla torsione ».

13. - L' influenza della tensione si manifesta anche quando non producendosi il precedente fenomeno, la torsione dà le curve caratteristiche a due cappi, per essere il campo sufficientemente intenso.

Con carichi tensori piccoli la curva del ciclo magnetoelastico è percorsa in modo che il ramo corrispondente alla detorsione è sempre inferiore a quello relativo alla torsione; per tensioni rilevanti avviene il rovescio: si ha un'inversione di segno della isteresi. Nel primo caso ciò̀ il cappio dalla parte positiva del campo $H_{1}$ è percorso nello stesso senso di quello degli indici d' un orologio, nel secondo invece in verso contrario. Dall' un caso all'altro si passa gradualmente, in modo che, con alcune tensioni, $i$ rami della torsione e della detorsione si tagliano formando due cappi nella regione positiva del campo $\mathrm{H}_{1}$ e due altri, ad essi praticamente simmetrici, nell'altra. Lo provano i dati della seguente tabella ottenuti col campo costante $\mathrm{H}_{1}=10.05$ unità.

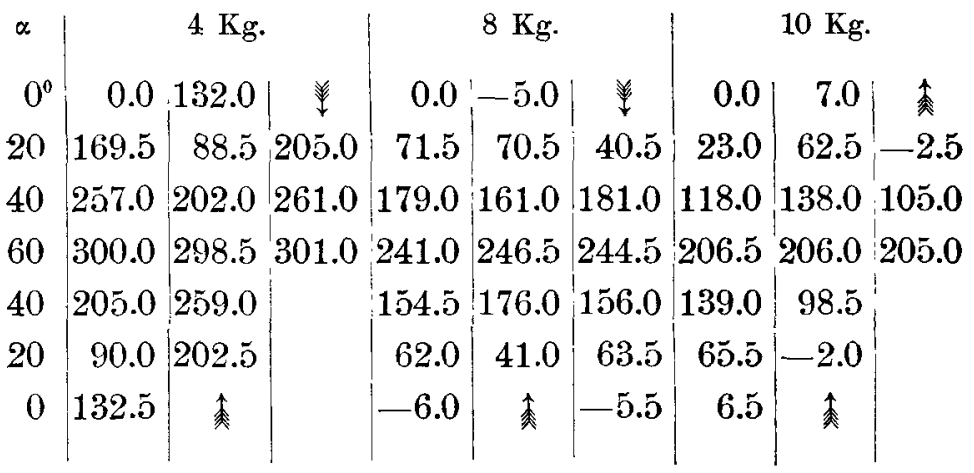


La fig. 3 è la rappresentazione grafica di questi risultati. Si scorge da essa come col crescer del carico il ciclo si accosti all' origine, poichè la prima torsione gencra aumenti di magnetizzazione che decreseono quando la tensione cresce.

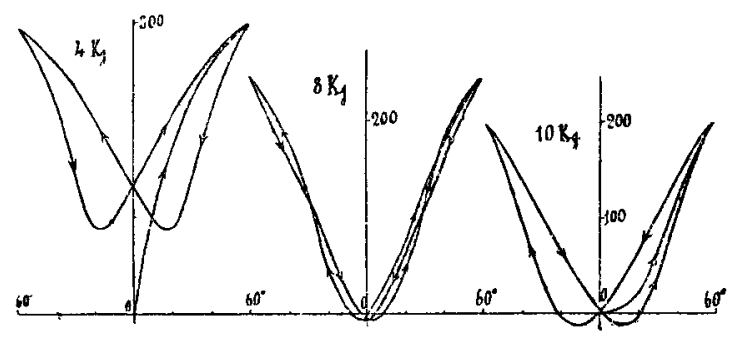

Fig. 3.

Se il campo magnetico è più debole del precedente questi fenomeni arvengono per carichi inferiori.

È d'avvertire però che tutto ciò dipende molto dallo stato elastico del filo che si esamina. Ho ottenuto i risultati precedenti studiando lo stesso filo con carichi crescenti di 2 in $2 \mathrm{Kg}$. Fili nuovi richiedono in generale tensioni maggiori e campi più intensi, o azioni elastiche ripetute, per presentare gli stessi fenomeni di uguale entità. L'incrudimento prodotto dalle deformazioni ha dunque sensibile influenza, come mostrerò poi direttamente in altro modo.

14. - Coi fili ciclizzati magneticamente fra i valori estremi $\mathrm{H}_{4}= \pm 12.2$ unita la torsione, con tensione da 2 a 12 Kg., produce effetti diversi, con lo stesso campo, a seconda del punto in cui la si comincia, nel senso che, arrestandosi ai punti del ciclo magnetico più prossimi all' asse $H_{1}$, essa provoca in generale variazioni del momento più grandi di quelle che genera iniziandola da punti più distanti, ottenendosi in quest' ultimo caso un ciclo magneto-elastico più prossimo all'asse stesso.

Ora, se si determina il ciclo magnetico e la curva di magnetizzazione reversibile del nichel ricotto sotto diverse tensioni si trova che, quando queste cominciano ad esser considerevoli, quella curva traversa ben presto il ciclo rimanendo 
per buona parte al di sopra di esso. Ciò risulta da questa tabella in eui, per brevita, sono riportati solo i punti del ciclo magnetico corrispondenti ai valori positivi di $\mathrm{H}_{1}$, e sono segnati nelle prime due colonne sotto i $\mathrm{Kg}$.; le terze comprendono i punti della curva anisteretica.

\begin{tabular}{|c|c|c|c|c|c|c|}
\hline $\mathrm{H}_{\mathrm{t}}$ & \multicolumn{3}{|c|}{$8 \mathrm{Kg}}$. & \multicolumn{3}{|c|}{$10 \mathrm{Kg}$. } \\
\hline 0.0 & -12.0 & $11 . \tilde{0}$ & 0.0 & 2.0 & $-1 . \bar{o}$ & 0.0 \\
\hline 1.0 & -13.0 & $9 . \check{5}$ & 10.0 & 0.0 & -3.0 & 5.0 \\
\hline 4.0 & $-11 . \tilde{5}$ & 7.5 & $21 . \overline{0}$ & -4.5 & -7.0 & 12.0 \\
\hline 7.0 & -7.0 & 6.0 & 28.0 & -9.0 & -11.0 & 19. \\
\hline .05 & 0.0 & 3.5 & 31.0 & -12.5 & -14.0 & 3.0 \\
\hline 12.2 & 5.5 & 㕛 & 32.0 & -15.5 & 南 & 25.0 \\
\hline
\end{tabular}

Col carico di $8 \mathrm{Kg}$. il ciclo è a isteresi positiva, ma con $10 \mathrm{Kg}$. essa diventa negativa e l'asse del ciclo cambia inclinazione.

Dunque anche " nel nichel ricotto, sufficientemente teso, i risultati diversi che con lo stesso campo magnetico si ottengono a seconda del punto del ciclo magnetico da cui ha principio la torsione, non posson più riferirsi alla curva di magnetizzazione anisteretica ».

15. - - La riduzione parziale d'isteresi magnetica operata dal campo alternato $\mathrm{H}_{z}$ cambia la forma dei cicli magnetoelastici in modo vario dipendente dall' intensità del campo costante $H_{1}$ e dalla tensione. Nella fig. 4 son rappresentati tre cicli ottenuti con $H_{4}=10.0 \tilde{s}$ e $H_{2}=20.1$ unità c. g. s. La I corrisponde al carico di $4 \mathrm{Kg}$., la II a quello di $8 \mathrm{Kg}$. e la III all'altro di $12 \mathrm{Kg}$. Al crescer della tensione aumentano le variazioni provocate dalla torsione, ciò che mostra che "la riduzione d'isteresi operata dallo stesso campo alternato diventa minore più grande $\grave{e}$ il carico tensore $"$.

Ma se il campo $\mathrm{H}_{2}$ è sufficientemente intenso $\left(\mathrm{H}_{2}=50.7\right.$ unita) tutte le variazioni diventano insignificanti per campi $\mathrm{H}_{1}$ deboli, mentre quando $\mathrm{H}_{1}=10.0 \tilde{\mathrm{a}}$ e $\mathrm{H}_{3}=50.7$ unità la 




Fig. 4.

torsione produce ancora dei piccoli mutamenti di magnetizzazione, come mostra la seguente tabella:

\begin{tabular}{|c|c|c|c|c|c|}
\hline & Kg. & Kg. & $\mathrm{Kg}$. & 12 & $\mathrm{Kg}$ \\
\hline & $0.0-2.0$ & $0.0-3.5$ & 0.0 & 0.0 & \\
\hline & $-2.0-3.0$ & $-3 . \bar{\delta}-4.0$ & $-2.0-1.0$ & 0.0 & \\
\hline & $-9.0-7.0$ & $-10.0-6$ & $-3.0-3.0$ & 0.0 & \\
\hline & $-12 . \tilde{0}-12.0$ & $-14.0-13.0$ & $-2.0-4.0$ & 0.0 & \\
\hline & $.5-7.5$ & $-7.0-10.0$ & $-2.0-4.0$ & 1.0 & \\
\hline & $-4.0-2.5$ & $-4.0-8.0$ & $-2.0-3.0$ & ธ..0 & \\
\hline & -1.0 & -2.0 & -1.0 & 4.0 & \\
\hline
\end{tabular}

Le variazioni che si osservano sono considerevolmente minori di quelle che hanno luogo nel solo campo costante $\mathrm{H}_{1}=10.05$ unità, ma mentre pei carichi minori si manifefestano come piccole diminuzioni, pei grandi si con servano ancora positive, sebbene in generale minori delle prime in valore assoluto. Ad ogni modo può dirsi che con campi alternati ancor più intensi esse forse finirebbero per scomparire.

16. - Dai risultati riferiti ai §§ 12 e 13 si deduce che, a seconda dello stato elastico del filo di nichel, la curva rappresentante le variazioni del momento nella detorsione può 
essere superiore (terza curva della Fig. 3) o inferiore (prima curva della Fig. 3) a quella relativa ai mutamenti dovuti alla torsione. Ho perciò ricercato se, anche con piccoli pesi traenti e campi magnetici costanti deboli, fosse possibile ottenere gli stessi fatti e produrre il fenomeno Nagaoka ma con cicli ad isteresi positiva, come appunto è succeduto (\$ 12) nel campo terrestre, ma sotto l'azione d' un forte carico $(10 \mathrm{Kg})$.

Partendo da un filo ben ricotto, con operazioni elastiche ripetute è possibile far variare gradatamente il suo stato elastico e stabilire perciò come variano i fenomeni magnetici che esso presenta nei successivi cambiamenti interni (incrudimento) per cui passa.

Un filo, ricotto al rosso vivo con una corrente di 24 a. per $600^{\mathrm{s}}$ e diminuita poi lentamente fino ad annullarla in $600^{\mathrm{s}}$, è assoggettato al carico di $2 \mathrm{Kg}$., smagnetizzato e torto sempre fra $\mathrm{i}$ valori estremi di $\pm 60^{\circ}$ nel solo campo terrestre. Il primo ciclo eseguito ha dato questi risultati :

\begin{tabular}{r|r|r}
$\alpha$ & & \\
$0^{\circ}$ & 0,0 & -10.5 \\
20 & 10.5 & 40.0 \\
40 & 66.0 & 108.5 \\
60 & 136.0 & 148.0 \\
40 & 92.5 & 108.0 \\
20 & 31.5 & $5 \check{5} .0$ \\
0 & 12.0 & 本
\end{tabular}

La curva che li rappresenta è costituita di rami che si inalzano da parti opposte dell'asse dell'intensità di magnetizzazione: è quindi del tipo di quelle che si ottengono ordinariamente con campi magnetici molto più intensi.

Ripetendo tre o quattro volte il ciclo elastico si nota che il ramo di curva a sinistra si abbassa verso la regione negativa dei momenti magnetici.

Si fanno allora due cicli completi di torsione sotto le tensioni di 4, 6 e $8 \mathrm{Kg}$. ottenendo le solite curve ad un cap- 
pio e ad isteresi negativa (Nagaoka) '). Riportato il carico a $2 \mathrm{Kg}$., il filo ha dato due cicli magneto-elastici rappresentati dalla I della Fig. 5: il secondo, molto lungo, ad isteresi negativa. Dopo la ripetizione delle operazioni precedenti, sotto la tensione di $2 \mathrm{Kg}$. si ottiene un ciclo simile a I, ma piu corto e un po' più stretto. Si fanno ancora due cicli di torsione sotto 4, 6 e $8 \mathrm{Kg}$., e, sempre col carico di $2 \mathrm{Kg}$., si trova che i rami delle curve di torsione si intrecciano verso le estremita; ma se si ripetono ancora due cicli di torsione a 4 e $6 \mathrm{Kg}$. e poi 10 a $8 \mathrm{Kg}$., ritornando a $2 \mathrm{Kg}$. si ha che quest' intrecciamento delle curve avviene all' incirca nell' origine, come mostra la II della Fig. ó. Ogni ciclo è ora costituito di due cappi, ma situati da parti opposte dei due assi e percorsi ognuno in senso inverso a quello degli indici d'un orologio.

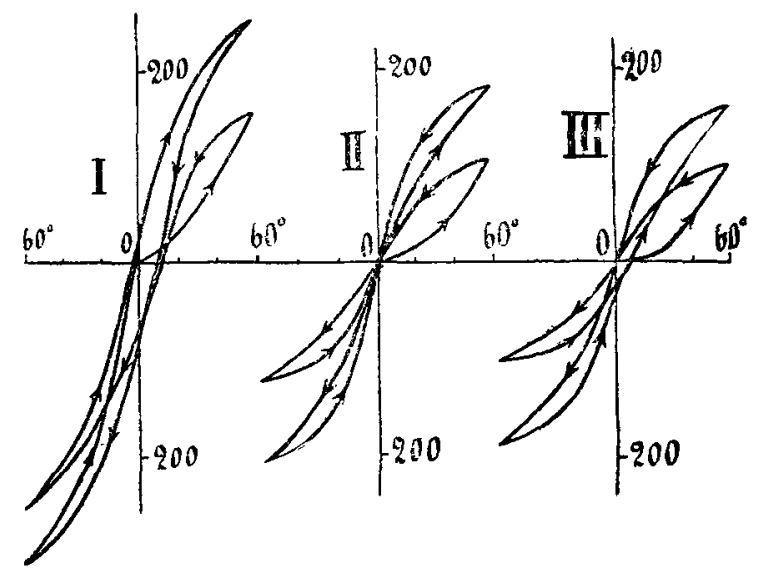

Fig. 5.

Ripetendo le ultime operazioni precedenti il ciclo magnetoelastico a $2 \mathrm{Kg}$. cambia e diventa come indica la III della Fig. 5. I cappi si sono staccati e il ciclo è ad isteresi positiva; si presenta, è vero, un po' stretto nel mezzo, ma con

1) Prima di cominciare ogni misura, in queste come in tutte le successive operazioni, il filo è sempre assoggettato a smagnetizzazione. 
ulteriori modificazioni elastiche si allarga per divenire quasi perfettamente simmetrico rispetto all' origine.

Si sono dunque ottenuti fin qui dei fenomeni simili a quelli che abbiamo veduto prodursi nei cicli costituiti da curve a due cappi con tensioni e campi assai più intensi : le tre curve della Fig. 5 corrispondono rispettivamente alle tre della Fig. 2.

Con la ripetizione reiterata di cicli sempre fra $i$ valori estremi di $\pm 60^{\circ}$, ma sotto tensioni diverse, esaminando di quando in quando il ciclo magneto-elastico con $2 \mathrm{Kg}$. si trova che esso diminuisce sempre più d'ampiezza, manifestandosi però una più rapida decrescenza nella parte situata nella regione dei momenti negativi. In ultimo ho ottenuto:

\begin{tabular}{|c|c|c|c|c|}
\hline$\alpha$ & \multicolumn{2}{|c|}{$1^{0}$ ciclo } & \multicolumn{2}{|c|}{$2^{0}$ ciclo } \\
\hline $0^{0}$ & 0.0 & 5.0 & 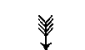 & 1.0 \\
\hline 20 & 1.0 & อ. 0 & 6.0 & 1.0 \\
\hline 40 & 5.0 & 6.0 & 10.0 & 1.0 \\
\hline 60 & $13 . \tilde{5}$ & 6.0 & $1 \tilde{5} .5$ & 1.0 \\
\hline 40 & 13.0 & 3.5 & 15.0 & 3.0 \\
\hline 20 & 11.0 & 3.0 & 13.0 & 5.0 \\
\hline 0 & 6.5 & 奏 & 8.5 & 委 \\
\hline
\end{tabular}

Il ciclo è ora molto stretto, prossimo all'asse del campo $\mathrm{H}_{1}$ e giace tutto nella regione positiva dei momenti magnetici : il fenomeno Nagaoka è scomparso.

Una serie ulteriore di cicli sotto forte tensione ha prodotto la rottura del filo.

I precedenti risultati mostrano quanto grande sia l'influenza che sui fenomeni magneto-elastici del nichel esercita lo stato elastico in cui esso si trova o è portato da deformazioni ${ }^{1}$ ), e permettono di concludere che " anche in campi

1) Risultati più complessi si ottengono esaminando $i$ mutamenti del ciclo magneto-elastico prodotto da torsioni bilaterali d'ampiezza costante, quando però si alterino i fili con torsioni di queste assai più grandi. 
magnetici deboli e con piccole tensioni il nichel può fornire cicli magneto-elastici ad un cappio ad isteresi positiva o negativa».

17. - Fili crudi. - Abbiamo veduto (\$ 6) che il ciclo magnetico fissato $\dot{e}$ in questi fili ad isteresi negativa, assai stretto e prossimo all'asse del campo, quando essi sono sotto la tensione di $4 \mathrm{Kg}$. mentre la curva anisteretica è molto elevata.

Crescendo il carico anche fino a $20 \mathrm{Kg}$. il ciclo resta sempre ad isteresi negativa, si avvicina ancora un po' all'asse del campo e la curva anisteretica si abbassa rimanendo sempre, per buona porzione, superiore al ciclo eccetto che per la tensione massima di $20 \mathrm{Kg}$. in cui lo sopravanza solo nei valori più elevati del campo $H_{1}$. Tutto ciò mostrano i seguenti resultati ove le prime due colonne sotto il numero dei $\mathrm{Kg}$. si riferiscono al ciclo magnetico fissato dalla parte dei valori positivi di $H_{1}$ e la terza alla curva di magnetizzazione anisteretica.

\begin{tabular}{|c|c|c|c|c|c|c|c|c|c|}
\hline $\mathbf{H}_{4}$ & \multicolumn{3}{|c|}{$10 \mathrm{Kg}$. } & \multicolumn{3}{|c|}{$16 \mathrm{Kg}}$. & \multicolumn{3}{|c|}{$20 \mathrm{Kg}}$. \\
\hline 0.0 & 13.0 & 1.0 & 0.0 & 6.0 & $|-7 . \check{0}|$ & 0.0 & 5.5 & $|--6.5|$ & 0.0 \\
\hline 1.0 & 17.5 & 7.0 & ๖઼1.ร & 11.5 & -2.5 & 7.0 & 10.0 & -3.5 & 5.0 \\
\hline 4.0 & 32.0 & 17.0 & 116.0 & 20.5 & $7 . \overline{\mathrm{s}}$ & 28.5 & 18.5 & $5 . \tilde{~}$ & 18.0 \\
\hline 7.0 & 41.5 & 30.0 & $139 . \check{~}$ & 28.5 & 18.0 & 37.0 & 26.0 & 14.5 & 26.5 \\
\hline 10.05 & 44.0 & 40.5 & 163.5 & 31.0 & 26.0 & 48.0 & $27 . \overline{5}$ & 21.0 & 35.0 \\
\hline 2.2 & 48.0 & 本 & 184.5 & 34.5 & 卖 & 60.5 & 28.5 & 委 & 45.0 \\
\hline
\end{tabular}

Ciclizzando magneticamente i fili, con la torsione si ottengono al solito, per lo stesso valore del campo $\mathrm{H}_{1}$, risultati diversi a seconda del punto del ciclo da cui si parte. Cosi con la tensione di $10 \mathrm{Kg}$. e il campo costante $\mathrm{H}_{1}=5.2$ unità, raggiunto da prima nel punto più alto del ciclo magnetico e poi in quello più basso rispetto all'asse $H_{1}$, ho avuto $i$ risultati scritti rispettivamente a sinistra e a destra della seguente tabella : 
VARIAZIONI MAGNETICHE ECC.

\begin{tabular}{|c|c|c|c|c|c|c|c|c|}
\hline & \multicolumn{2}{|c|}{$1^{0}$ eiclo } & \multicolumn{2}{|c|}{$2^{\circ}$ ciclo } & \multicolumn{2}{|c|}{$1^{0}$ ciclo } & \multicolumn{2}{|c|}{$2^{\circ}$ ciclo } \\
\hline $0^{\circ}$ & 0.0 & 22.0 & 䜌 & 31.0 & 0.0 & 32.0 & 呈 & 42.0 \\
\hline & 0.0 & 23.0 & 21.0 & 32.0 & 4.5 & 32.0 & 32.0 & 43.5 \\
\hline & $3 . \tilde{5}$ & 23.0 & 22.0 & 33.0 & 8.0 & 33.0 & 33.0 & 44.0 \\
\hline 60 & 10.0 & 24.0 & 25.5 & 33.5 & 16.5 & $32 . \tilde{x}$ & 37.5 & 44.5 \\
\hline 40 & 11.0 & $16 . \check{~}$ & 26.0 & 29.5 & 17.0 & 25.5 & 37.0 & 40.0 \\
\hline & 11.0 & 13.0 & 26.0 & 27.0 & 18.0 & 21.0 & 37.0 & 39.0 \\
\hline O & 12.0 & 点 & 26.0 & 乘 & 19.5 & 㤐 & 38.0 & 奏 \\
\hline
\end{tabular}

Nel primo càso gli aumenti di magnetizzazione prodottí dalla torsione sono minori che nel secondo; nell'uno le curve. descritte dal punto rappresentativo della magnetizzazione iniziale sono più accoste fra loro e più prossime all' asse del. campo che nell'altro caso.

Anche qui gli effetti non son più da riferirsi alla curva. di magnetizzazione anisteretica.

18. Abbiamo veduto che i fenomeni presentati dal nichel crudo, e anche quelli offerti dal ricotto sottoposto a tensioni e campi non troppo piccoli, son tali che in generale non posson più interpretarsi riportandosi all'ordinaria curva di magnetizzazione anisteretica, mentre la considerazione di questa curva - come per primo ha rilevato il Maurain ') permette di classificare nettamente dei fenomeni molto vari e complessi presentati dal ferro crudo e quindi anche di prevederli quando sien note le condizioni iniziali.

La questione merita un esame più minuto e preciso di quello che potrei fare ora: in particolare è necessario conoscere le altre curve di magnetizzazione reversibile che possono aversi col campo alternato oltre quella considerata dal Maurain. In altra occasione ritornerò su quest'argomento che sto studiando.

19. Dall'insieme delle esperienze che ho fatto, nei limiti e condizioni in cui ho esperimentato, posson trarsi varie conclusioni.

1) Journal de Phys. 1907. 
Trazione. - $1^{0}$ L'influenza esercitata dalla trazione sul momento magnetico longitudinale del nichel dipende molto dal grado di ricottura, cioè dalla temperatura a cui si porta, dalla durata della sua azione e di quella del raffreddamento.

$2^{0}$ Nei fili ricotti al rosso vivo, sempre nello stesso modo, in campo magnetico costante $\mathrm{H}_{1}$ la trazione:

a) Lungo la curva di prima magnetizzazione produce una diminuzione del momento longitudinale e i cicli magnetoelastici si fissano spostandosi verso la curva anisteretica.

b) Su questa curva provoca diminuzioni delle precedenti più grandi; i cicli hanno forma più allungata e si fissano per intero al di sotto dell'asse del campo.

c) Nei fili magneticamente ciclizzati, in causa della riduzione $d$ 'isteresi magnetica che produce, genera da principio fenomeni vari dipendenti dall' intensità del campo. Considerando allora gli effetti delle trazioni successive alla prima risulta che "nei fili ciclizzati la tensione produce sempre diminuzione di magnetizzazione e il rilasciamento sempre aumento quando il campo costante $\mathrm{H}_{1}$ non è troppo debole (di 1 unità o più), mentre in campi poco intensi (campo terrestre) succede lo stesso se si parte da punti del ciclo magnetico posti al di sopra della curva di magnetizzazione reversibile, ed avviene il rovescio movendosi, per lo stesso valore del campo, da punti situati inferiormente a quella curva ». In ogni caso verso di essa si sposta il ciclo magneto-elastico.

$3^{0}$ I fili crudi di nichel presentano un ciclo magnetico molto stretto e ad isteresi negativa; la curva di magnetizzazione anisteretica lo traversa in una regione limitatissima, prossima cioè ad $\mathrm{H}_{1}=0$, e lo sopravanza. Risulta che la trazione :

a) Lungo la curva di prima magnetizzazione produce diminuzioni molto più piccole di quelle che genera nei fili ricotti; $\mathbf{i}$ cicli magneto-elastici si fissano per intero al di sotto dell' ascissa $H_{1}$ se il campo è debole (1 unità o meno), mentre se $\mathrm{H}_{4}$ è più intenso essi tagliano l'asse $\mathrm{H}_{4}$.

b) Lungo la curva di magnetizzazione anisteretica genera diminuzioni di magnetizzazione maggiori di quelle che si hanno nel caso precedente e $\mathrm{i}$ cicli si fissano al di sotto del- 
l'asse $H_{1}$ comprendendo un'area sensibilmente maggiore di quella che hanno, a pari condizioni, nel caso a).

c) Nei fili magneticamente ciclizzati seguita a produrre effetti diversi a seconda del punto del ciclo magnetico a cui, per lo stesso valore del campo, ci si arresta. Data la forma del ciclo magnetico e quella della curva anisteretica non è quindi più possibile riportare i fatti osservati a quella curva. $4^{0}$ Se al campo $H_{1}$ si sorrappone l'altro alternato $H_{2}$, quando questo non ha intensità sufficiente a ridurre completamente l' isteresi magnetica, i fenomeni prodotti dalla trazione, tanto nei fili ricotti che in quelli crudi, sono molto vari e complessi. In generale le variazioni di magnetizzazione si attenuano e ove l'intensità di $\mathrm{H}_{2}$ sia molto grande rispetto a quella di $\mathrm{H}_{1}$ la trazione non ha più che un' azione trascurabile.

Torsione. $-1^{0}$ Anche gli effetti prodotti dalla torsione variano col grado di ricottura dei fili.

$2^{\circ}$ Con campo magnetico debole (inferiore a 1 unità) e piccola tensione $\left(63.7 \mathrm{Kg}\right.$. per $\left.\mathrm{cm}^{2}\right)$ nei fili ricotti nel solito modo la torsione:

a) Produce il fenomeno Nagaoka : il ciclo magneto-elastico è ad isteresi negativa.

b) Iniziata da punti del ciclo posti al di sopra o al di sotto della curva reversibile, il ciclo si trova spostato sempre verso quella curva, ma la prima torsione genera un aumento di magnetizzazione nel primo caso, una diminuzione nel secondo.

c) Pù destare magnetizzazioni grandissime anche in campi molto deboli (campo terrestre) quando raggiunga il valore di 4 o $5^{0}$ per $\mathrm{cm}$.

$3^{0}$ In campo magnetico costante più intenso $(1$ o più unità) la torsione :

a) Lungo la curva di prima magnetizzazione genera aumenti di magnetizzazione e si hanno dei cicli magneto-elastici della nota forma a due cappi che si fissano spostandosi verso la curva anisteretica.

b) Su questa dà dei cicli che si fissano verso l'origine assai più dei precedenti. 
c) Nei fili magneticamente ciclizzati dà, per lo stesso. campo, dei cicli magneto-elastici spostati sempre verso la curva anisteretica.

$4^{0}$ Sotto l'azione del campo alternato $\mathrm{H}_{2}$ sufficientemente intenso $\left(\mathrm{H}_{3}=50.7\right.$ unita $)$ tutte le precedenti variazioni del momento magnetico risultano insensibili, o si convertono in piccole diminuzioni di magnetizzazione, quando il campo costante ha un valore considerevole $\left(H_{1}=10.05\right.$ unità).

$5^{0} \mathrm{Al}$ crescer del carico traente (da 63.7 a $382 \mathrm{Kg}$. per $\mathrm{cm}^{2}$.):

a) Il ciclo magnetico diminuisce di area accostandosi all' asse del campo e finisce per cambiare orientazione presentando la parte dei momenti positivi dal lato negativo dell' asse del campo $\mathrm{H}_{1}$; esso allora è molto stretto, prossimo all'asse e ad isteresi negativa.

b) La curva di magnetizzazione anisteretica si accosta all' asse del campo $H_{1}$, ma meno rapidamente del ciclo magnetico in guisa che finisce per rimanere quasi tutta a questo superiore.

c) In campi deboli (campo terrestre) il ciclo magnetico elastico si conserva della forma ad un sol cappio, diminuisce di area accostandosi all'asse del campo e finisce per cambiare orientazione come nel caso a): esso è allora stretto, prossimo all'asse e ad isteresi positiva mentre in principio questa è negativa.

Il rincrudimento prodotto dalla deformazione permanente (allungamento di 1.5 a $2 \%$ ) dovuta al massimo carico genera. qui, come in a), un'inversione del segno dell' isteresi magnetica e un' orientazione diversa dal eiclo.

d) In campi più intensi (maggiori di 2 o 3 unita) il ciclo magneto-elastico ha da prima la forma tipica a due cappi, poi i rami delle curve relativi alla torsione e detorsione si tagliano e infine ritorna alla forma a due cappi; è allora più prossimo all'asse del campo, ma presenta isteresi inversa alla primitiva (Fig. 3).

e) La torsione continua a produrre effetti diversi a seconda del punto del ciclo magnetico da cui la si comincia, sia questo a isteresi positiva o negativa, e quindi i fenomeni non 
son più, in generale, da riferirsi all'ordinaria curva anisteretica.

f) La riduzione parziale $\mathrm{d}$ ' isteresi operata dallo stesso campo alternato $\mathrm{H}_{2}$ non molto intenso, sovrapposto ad un dato $\mathrm{H}_{1}$ costante, diminuisce : i cicli magneto elastici variano molto di forma e dimensioni col mutare di $\mathrm{H}_{1}$ e $\mathrm{H}_{2}$.

g) Le piccole diminuzioni di magnetizzazione che si verificano da prima ( $\S 15)$ in un campo variabile intenso $\left(H_{1}=10.05\right.$ e $H_{2}=50.7$ unità) finiscono per rimanere degli aumenti ancor più piccoli.

$6^{\circ}$ Alterando lo stato d' un filo ben ricotto con torsioni ripetute la forma del ciclo magneto-elastico, che si ottiene nel campo terrestre e sotto lieve tensione $\left(63.7 \mathrm{Kg}\right.$. per $\left.\mathrm{cm}^{2}\right)$, varia moltissimo. Da una curva a due rami, innalzantisi da parti opposte dell'asse dell'intensità di magnetizzazione, si passa gradatamente ai cicli del Nagaoka a isteresi negativa; i rami delle curve di torsione e detorsione poi s' intrecciano, fig. 5, finalmente si separano per prodursi dei cicli ad un cappio ma ad isteresi positiva. In ultimo il ciclo diventa molto stretto, prossimo all'asse del campo e giacente tutto nella regione positiva dei momenti : il fenomeno Nagaoka scompare.

$7^{\circ}$ La torsione e la detorsione producono nei fili crudi di nichel un graduale aumento di magnetizzazione; ma anche questo scompare se al campo costante si sorrappone quello alternato intenso.

I diversi effetti che una stessa torsione può produrre in fili crudi magneticamente ciclizzati non posson più riferirsi alla curva anisteretica e si annullano quando $\mathrm{H}_{2}$ riduca completamente l'isteresi magnetica.

20. - Tutto sommato può dirsi che i fenomeni magnetoelastici presentati dal nichel dipendono moltissimo dallo stato elastico in cui si trova e che, entro i limiti delle esperienze eseguite, quando il campo è sufficiente a ridurre l'isteresi magnetica le azioni elastiche hanno effetto trascurabile sul momento magnetico longitudinale del nichel.

L' eliminazione della isteresi magnetica, operata dal campo alternato, $\Theta$ dalle vibrazioni longitudinali che questo genera nel nichel per magnetostrizione, porta dunque a risultati molto 
semplici. Ma non è escluso, per ora, ehe ciò non sia pur dor vuto ad altra causa. È noto infatti ') che, almeno nel ferro, il campo magnetico costante e le oscillazioni elettriche riducono sensibilmente la isteresi elastica per trazione. È verosimile che un tale effetto sulle varie deformazioni del nichel $\mathrm{e}$ del ferro lo abbia pure il campo alternato $\mathrm{H}_{2}$. Ma la natura delle esperienze che fin qui ho fatto non permette alcuna affermazione in proposito. E necessario quindi ch'io ricerchi se alla semplicizzazione dei risultati possa concorrere anche una probabile riduzione d'isteresi elastica operata dal campo alternato.

Napoli, dicembre 1910.

1) Grimaldi e Accolla. * Influenza delle scariche oscillatorie e del magnetismo sull'isteresi elastica del ferro per trazione ". Atti Acc. Gioe. nia. S. 5, Vol. II. 